Article

\title{
Initial Boundary Value Problems of Semi-Linear Sub-Diffusion with Gradient Terms
}

\author{
Yabing Gao * $\mathbb{D}$ and Yongxiang Li \\ Department of Mathematics, Northwest Normal University, Lanzhou 730070, China; liyxnwnu@163.com \\ * Correspondence: gyabing1610@163.com
}

Received: 6 September 2020; Accepted: 24 September 2020; Published: 27 September 2020

\begin{abstract}
We consider the existence and uniqueness of the saturated classical solutions and the positive classical solutions to initial boundary value problems of semi-linear sub-diffusion with gradient terms. Applying this to the fractional power of the sectorial operator theory and the imbedding theory in the interpolation spaces, where the nonlinear term satisfies more general conditions, we obtain the existence and uniqueness of the saturated classical solutions. The results obtained generalize the recent conclusions on this topic. Finally, an example is given to illustrate the feasibility of our main results.
\end{abstract}

Keywords: fractional partial differential equations; gradient terms; classical solutions; fractional power of sectorial operator

\section{Introduction}

At present, the fractional differential equations have been proved to be valuable tools in the investigation of many phenomena in such areas as physics, chemistry, fractal, economy, and engineering. It has been found that the differential equations involving the fractional derivative are more realistic to describe many phenomena in practical cases than those of an integer order.

During the last decade, the partial differential equations with time fractional derivatives have increasingly attracted the attention of researchers both in pure and applied mathematics, because they can be taken as mathematical models of complex systems which exhibit anomalous diffusion. Evidently, fractional partial differential equations are apt to describe diffusive motions that cannot be modeled as standard Brownian ones (described by heat equation or more general parabolic equations), where the mean square displacement of a diffusive particle is a linear function of time $t$. The signature of an anomalous diffusion of this kind is that mean square displacement of the diffusing species $\left\langle(\Delta x)^{2}\right\rangle$ scales as a nonlinear power law in time $t$, that is, $\left\langle(\Delta x)^{2}\right\rangle \sim K_{\gamma} t^{\gamma}$, where $K_{\gamma}$ is a constant. When $\gamma \in(0,1)$, this is referred to as sub-diffusion; when $\gamma>1$, this is referred to as supdiffusion.

The semi-linear fractional parabolic partial differential equations have been discussed by too many authors. In 2014, Chen et al. [1] considered the initial value problem of fractional semi-linear evolution equations with non-compact semigroups in Banach spaces $E$

$$
\left\{\begin{array}{l}
{ }^{C} D_{t}^{\gamma} u(t)+A u(t)=f(t, u(t)), \quad t \in J=[0, a], \\
u(0)=u_{0},
\end{array}\right.
$$

where ${ }^{C} D_{t}^{\gamma}$ is the Caputo fractional derivative of order $\gamma \in(0,1), A: D(A) \subset E \rightarrow E$ is a closed linear operator and $-A$ generates a uniformly bounded $C_{0}-\operatorname{semigroup} T(t)(t \geq 0)$ in $E, f \in C(J \times E \times E, E)$ is a continuous nonlinear mapping, $u_{0} \in E$. The existence of saturated mild solutions and global mild solutions is obtained under weaker conditions. Authors [2] are concerned with non-local problems for fractional evolution equations with mixed monotone non-local terms of the form 


$$
\left\{\begin{array}{l}
{ }^{C} D_{t}^{\gamma} u(t)+A u(t)=f(t, u(t), u(t)), \quad t \in J=[0, a], \\
u(0)=g(u, u),
\end{array}\right.
$$

where $E$ is an infinite-dimensional Banach space, ${ }^{C} D_{t}^{\gamma}$ is the Caputo fractional derivative of order $\gamma \in(0,1), A: D(A) \subset E \rightarrow E$ is a closed linear operator and $-A$ generates a uniformly bounded $C_{0}-$ semigroup $T(t)(t \geq 0)$ in $E, f \in C(J \times E \times E, E)$, and $g$ is an appropriate continuous function so that it constitutes a non-local condition. They construct a new monotone iterative method for non-local problems of fractional evolution equations with mixed monotone non-local terms and obtain the existence of coupled extremal mild L-quasi-solutions and the mild solution between them. In 2017, Mu et al. [3] investigated some initial-boundary value problems for time-fractional diffusion equations, and some interesting versions of maximal and spatial regularity criteria are discussed. In 2020, Chen et al. [4] discussed the Cauchy problem to a class of nonlinear time-fractional non-autonomous integro-differential evolution equations of mixed type via a measure of non-compactness in infinite-dimensional Banach spaces; further, Reference [5] is concerned with the existence of mild solutions, as well as approximate controllability for a class of fractional evolution equations with non-local conditions in Banach spaces. In the Reference [6], El-Borai and Debbouche established the existence and uniqueness of local mild, then local classical solutions of a class of nonlinear fractional integro-differential equations in Banach space with analytic semigroups. Luchko [7] first obtained the maximum principle for fractional parabolic partial differential equations. In 2020, Zhang et al. [8] discussed a class of fractional retarded differential equations involving mixed non-local plus local initial conditions.

On the other hand, we noticed that among the previous researchers, most of the researchers focus on the case when the nonlinear terms are without gradient terms, which means that the above authors have obtained results which cannot be used when the nonlinear terms are with gradient terms. Motivated by the above mentioned aspects, in this paper, we will investigate the existence and uniqueness of the saturated classical solutions to the following problems

$$
D_{t}^{\gamma} u(x, t)+A(x, D) u(x, t)=f(x, t, u(x, t), \nabla u(x, t)), \quad(x, t) \in \Omega \times(0,+\infty),
$$

subject to the boundary condition

$$
\left.u\right|_{\partial \Omega}=0, \quad t \in(0,+\infty),
$$

and the initial condition

$$
u(x, 0)=\varphi(x), \quad x \in \bar{\Omega},
$$

where $\Omega$ is a bounded domain in $\mathbb{R}^{N}(N \geq 1)$ with smooth boundary $\partial \Omega, \gamma \in(0,1)$ is a real number, and the symbol $D_{t}^{\gamma}$ represents the regularized Caputo fractional derivative of order $\gamma$ with respect to the time variable. The unknown function $u=u(x, t): \Omega \times(0,+\infty) \rightarrow \mathbb{R}, \nabla$ stands for the gradient operator with respect to the spatial variable, and the $\varphi(x)$ is a given function. The nonlinear term $f: \bar{\Omega} \times \mathbb{R}^{+} \times \mathbb{R} \times \mathbb{R}^{N} \rightarrow \mathbb{R}$ is continuous and

$$
A(x, D) u:=-\sum_{i, j=1}^{N} \frac{\partial}{\partial x_{i}}\left(a_{i j}(x) \frac{\partial u}{\partial x_{j}}\right)+a_{0}(x) u
$$

is a uniformly elliptic differential operator of divergence type in $\bar{\Omega}$ with the coefficients $a_{i j} \in$ $C^{1+\sigma}(\bar{\Omega})(i, j=1,2, \cdots, N)$ and $a_{0} \in C^{\sigma}(\bar{\Omega})$ and $a_{0}(x) \geq 0$ on $\bar{\Omega}$. That is, $\left[a_{i j}(x)\right]_{N \times N}$ is a positive definite symmetric matrix for every $x \in \bar{\Omega}$ and there exists a constant $\varrho>0$, such that

$$
\sum_{i, j=1}^{N} a_{i j}(x) \zeta_{i} \zeta_{j} \geq \varrho|\zeta|^{2}, \quad \forall \zeta=\left(\zeta_{1}, \zeta_{2}, \cdots, \zeta_{N}\right) \in \mathbb{R}^{N}, x \in \bar{\Omega}
$$


The rest of this paper is organized as follows. In Section 2, we present some preliminaries on fractional calculus, the definitions and properties of the fractional powers about sectorial operator, interpolation spaces, and some lemmas, which will be used in the proof of our main result. Section 3 states and proves the saturated classical solutions and the positive classical solutions for the initial value problem of semi-linear sub-diffusion with gradient terms (1)-(3). In Section 4, an example is given to illustrate the feasibility of our main results. In Section 5, we present our future work.

\section{Preliminaries}

Let $X$ be a Banach space with norm $\|\cdot\|$. We denote by $C([0, T], X)$ the Banach space of all continuous functions from interval $[0, T]$ into $X$ equipped with the norm $\|u\|_{C}=\max _{t \in[0, T]}\|u(t)\|$. Assume $u:[0, \infty) \rightarrow X$. The fractional integral of order $\gamma>0$ for the function $u$ is defined as

$$
J_{t}^{\gamma} u=\frac{1}{\Gamma(\gamma)} \int_{0}^{t}(t-s)^{\gamma-1} u(s) d s, \quad t>0 .
$$

The standard Caputo fractional derivative of order $0<\gamma \leq 1$ for the function $u$ is defined by

$$
{ }^{C} D_{t}^{\gamma} u(t)=\frac{1}{\Gamma(1-\gamma)} \int_{0}^{t}(t-s)^{-\gamma} f^{\prime}(s) d s, \quad t>0 .
$$

Further, $D_{t}^{\gamma}$ represents the regularized Caputo fractional derivative of order $0<\gamma \leq 1$ for the function $u$ and is defined by

$$
D_{t}^{\gamma} u(t)=\frac{d}{d t}\left[J_{t}^{1-\gamma}(u(t)-u(0))\right], \quad t>0 .
$$

For $u:[0, \infty) \times \mathbb{R}^{N} \rightarrow \mathbb{R}$, the regularized Caputo time-fractional derivative of the function $u$ can generally be written as $D_{t}^{\gamma}$

$$
D_{t}^{\gamma} u(x, t)=\frac{d}{d t}\left[J_{t}^{1-\gamma}(u(x, t)-u(x, 0))\right]=\frac{1}{\Gamma(1-\gamma)} \frac{\partial}{\partial t} \int_{0}^{t} \frac{u(x, s)-u(x, 0)}{(t-s)^{\gamma}} d s, \quad t>0 .
$$

For more insight into the topic, see Kilbas et al. [9].

The Hölder space with exponent $\sigma$ from $\bar{\Omega}$ into $X$ is denoted by $C^{m+\sigma}(\bar{\Omega}, X)(m=0,1,2 \ldots ; 0<$ $\sigma<1)$. For details of the Hölder space, the usual $p$ th power integrable function spaces: $L^{p}(\Omega)(p \geq 1)$ and the usual Sobolev spaces: $W^{m, p}(\Omega)$, we refer to Adams [10], Evans [11], and Henry [12] which are denoted by $B(X)$ the Banach space of all linear bounded operators in $X$ endowed with the topology defined by the operator norm. The $X_{1} \hookrightarrow X_{2}$ means that the Banach space $X_{1}$ is continuously embedded in the Banach space $X_{2}$.

We introduce fractional Hölder spaces:

$$
F C^{k+\sigma, \frac{k+\sigma}{2} \gamma}(\bar{\Omega} \times[0, T]), \quad \gamma, \sigma \in(0,1)
$$

for an arbitrary fixed time $T>0$ with norms

$$
\begin{gathered}
\|u\|_{F C^{k+\sigma, \frac{k+\sigma}{2} \gamma(\bar{\Omega} \times[0, T])}}=\|u\|_{C\left([0, T], C^{k+\sigma}(\bar{\Omega})\right)}+\sum_{|i|=0}^{k}\left\langle D_{x}^{i} u\right\rangle_{t, \Omega \times(0, T)}^{\left(\frac{k+\sigma-|i|}{2} \gamma\right)}, \quad k=0,1 ; \\
\|u\|_{F C^{2+\sigma, \frac{2+\sigma}{2} \gamma}(\bar{\Omega} \times[0, T])}=\|u\|_{C\left([0, T], C^{2+\sigma}(\bar{\Omega})\right)}+\left\|D_{t}^{\gamma} u\right\|_{F C^{\sigma, \frac{\sigma}{2} \gamma}(\bar{\Omega} \times[0, T])}+\sum_{|i|=1}^{2}\left\langle D_{x}^{i} u\right\rangle_{t, \Omega \times(0, T)}^{\left(\frac{2+\sigma-|i|}{2} \gamma\right)},
\end{gathered}
$$


where

$$
\left\langle D_{x}^{i} u\right\rangle_{t, \Omega \times(0, T)}^{\left(\sigma^{*}\right)}=\sup _{\bar{\Omega} \times[0, T]} \frac{\left|D_{x}^{i} u(x, t)-D_{x}^{i} u(x, \bar{t})\right|}{|t-\bar{t}|^{*}}, \quad t \neq \bar{t}, \quad \sigma^{*} \in(0,1), \quad i=0,1,2 .
$$

It is obvious fact that

$$
\begin{gathered}
F C^{\sigma, \frac{\sigma}{2} \gamma}(\bar{\Omega} \times[0, T])=C^{\sigma, \frac{\sigma}{2} \gamma}(\bar{\Omega} \times[0, T]) ; \\
F C^{2+\sigma_{2}}, \frac{2+\sigma_{2}}{2} \gamma(\bar{\Omega} \times[0, T]) \hookrightarrow F C^{2+\sigma_{1}}, \frac{2+\sigma_{1}}{2} \gamma(\bar{\Omega} \times[0, T]), \quad 0<\sigma_{1}<\sigma_{2}<1 .
\end{gathered}
$$

For details of the fractional Hölder spaces, see [13].

In the latter discussion, we denote

$$
X=L^{p}(\Omega)(p \geq 2)
$$

and define the linear operator

$$
A u=A(x, D) u
$$

with the domain

$$
D(A)=W^{2, p}(\Omega) \cap W_{0}^{1, p}(\Omega) .
$$

It is well-known that $A$ is a positive definite self-adjoint operator and $-A$ generates an analytic operator semigroup $T(t)(t \geq 0)$ in $X$ (see ([14], Chapter 2, Sections 5 and 6)). Let $\lambda_{1}$ be the first eigenvalue of the operator $A$ under the Dirichlet boundary condition $\left.u\right|_{\partial \Omega}=0$. For any $0<\delta<\lambda_{1}$, there is a constant $M \geq 1$, such that

$$
\|T(t)\| \leq M e^{-\delta t}, \quad t \geq 0 .
$$

Clearly, $A$ is a sectorial operator. It is also well-known that each sectorial operator can define fractional powers. Next, we recall some concepts and conclusions on the fractional powers of $A$. For $\alpha>0, A^{-\alpha}$ is defined by

$$
A^{-\alpha}=\frac{1}{\Gamma(\alpha)} \int_{0}^{\infty} t^{\alpha-1} T(t) d t
$$

where $T(t)(t \geq 0)$ is an analytic operator semigroup, which is generated by $-A$. $A^{-\alpha} \in B(X)$ is injective, and $A^{\alpha}$ can be defined by $A^{\alpha}=\left(A^{-\alpha}\right)^{-1}$ with the domain $D\left(A^{\alpha}\right)=A^{-\alpha}(X)$. For $\alpha=0$, let $A^{\alpha}=I$. We endow a norm $\|x\|_{\alpha}=\left\|A^{\alpha} x\right\|$ to $D\left(A^{\alpha}\right)$. Since $A^{\alpha}$ is a closed linear operator, it follows that $\left(D\left(A^{\alpha}\right),\|\cdot\|_{\alpha}\right)$ is a Banach space. We denote by $X_{\alpha}$ the Banach space $\left(D\left(A^{\alpha}\right),\|\cdot\|_{\alpha}\right)$. Denote $X_{0}=X, X_{1}=D(A)$, then

$$
X_{1} \subset X_{\alpha} \subset X_{0}=X, \quad 0<\alpha<1 .
$$

The space $X_{\alpha}$ is named the interpolation space between $X_{0}$ and $X_{1}$ or the fractional power space of the sectorial operator $A$. For $0 \leq \alpha_{1}<\alpha_{2}, X_{\alpha_{2}}$ is bounded and embedded into $X_{\alpha_{1}}$, and the embedding $X_{\alpha_{2}} \hookrightarrow X_{\alpha_{1}}$ is compact when the operator $A$ exists for compact resolvent. For details of the properties of the fractional powers about the sectorial operator, refer to Carracedo and Alix [15], Henry [12], and Pazy [14].

Lemma 1 ([14]). Let $0 \leq \alpha_{1}<\alpha_{2} \leq 1$ and $X_{\alpha_{1}}, X_{\alpha_{2}}$ be the fractional power space of the sectorial operator $A$. These possess a constant $\bar{c}=\bar{c}\left(\alpha_{1}, \alpha_{2}\right)$, such that for every $x \in X_{\alpha_{2}}$ and $\varepsilon>0$, we have

$$
\|x\|_{\alpha_{1}} \leq \bar{c}\left(\varepsilon^{\frac{\alpha_{1}}{\alpha_{2}}}\|x\|+\varepsilon^{\frac{\alpha_{1}}{\alpha_{2}}-1}|| x \|_{\alpha_{2}}\right),
$$


and choosing $\varepsilon=\frac{\|x\|_{\alpha_{2}}}{\|x\|}$, we have

$$
\|x\|_{\alpha_{1}} \leq 2 \bar{c}|| x\left\|_{\alpha_{2}}^{\frac{\alpha_{1}}{\alpha_{2}}} \cdot\right\| x \|^{1-\frac{\alpha_{1}}{\alpha_{2}}}
$$

The interpolation space $X_{\alpha}$ and the Hölder space satisfies the following relationships:

Lemma 2 ([12]). Suppose $\Omega$ be an open, bounded domain in $\mathbb{R}^{N}$ with $C^{2}$-smooth boundary $\partial \Omega$. Then,

(1) $X_{\alpha}$ is compactly embedded in $C^{1+\sigma}(\bar{\Omega})$ if $0 \leq \sigma<2 \alpha-1-\frac{N}{p}$ for $\frac{1}{2}+\frac{N}{2 p}<\alpha \leq 1$ when $p>N$.

(2) $X_{\alpha}$ is compactly embedded in $C^{\sigma}(\bar{\Omega})$ if $0 \leq \sigma<2 \alpha-\frac{N}{p}$ for $\frac{N}{2 p}<\alpha \leq 1$ when $p>\frac{N}{2}$.

We introduce the following Schauder theory of the linear fractional parabolic partial differential equations.

Lemma 3 ([13]). Let $\Omega$ be an open, bounded domain in $\mathbb{R}^{N}$ with $C^{2+\mu}$-smooth boundary $\partial \Omega$. For any fixed $T>0$, the functions $\varphi(x) \in C^{2+\mu}(\bar{\Omega})$ and $h(x, t) \in F C^{\mu, \frac{\mu \gamma}{2}}(\bar{\Omega} \times[0, T])$ and the compatibility conditions

$$
\varphi=0 \quad \text { and } \quad A(x, D) \varphi=h(x, 0), \quad x \in \partial \Omega .
$$

is hold. Then the following initial boundary value problem of linear fractional parabolic type (LFP)

$$
\left\{\begin{array}{l}
D_{t}^{\gamma} u(x, t)+A(x, D) u(x, t)=h(x, t), \quad(x, t) \in \Omega \times(0,+\infty), \\
\left.u\right|_{\partial \Omega}=0, \\
u(x, 0)=\varphi(x), \quad x \in \bar{\Omega},
\end{array}\right.
$$

possesses a unique classical solution:

$$
u \in F C^{2+\mu, \frac{2+\mu}{2} \gamma}(\bar{\Omega} \times[0, T])
$$

Denote

$$
C([0, T] \times \bar{\Omega})=C([0, T], C(\bar{\Omega})) .
$$

For any $t \in[0, T]$, let $h(t)=h(\cdot, t)$. The continuous embedding $C^{\mu}(\bar{\Omega}) \hookrightarrow X$ implies that the map $h:[0, T] \rightarrow X$ is continuous. Now, we consider the initial value problem of the linear evolution equations in $X$ (LEE)

$$
\left\{\begin{array}{l}
D_{t}^{\gamma} u(t)+A u(t)=h(t), \quad t \in[0, T] \\
u(0)=\varphi \in X_{\alpha} .
\end{array}\right.
$$

Definition 1. A function $u \in C([0, T], X)$ is said to be a mild solution of the $L E E$ (5) if it satisfies

$$
u(t)=S_{\gamma}(t) \varphi+\int_{0}^{t}(t-s)^{\gamma-1} P_{\gamma}(t-s) h(s) d s:=H(\varphi, h),
$$

where $h \in C([0, T], X)$ and

$$
\begin{gathered}
S_{\gamma}(t)=\int_{0}^{\infty} \zeta_{\gamma}(s) T\left(t^{\gamma} s\right) d s, \quad P_{\gamma}(t)=\gamma \int_{0}^{\infty} s \zeta_{\gamma}(s) T\left(t^{\gamma} s\right) d s, \\
\zeta_{\gamma}(s)=\frac{1}{\pi \gamma} \sum_{n=1}^{\infty}(-s)^{n-1} \frac{\Gamma(n \gamma+1)}{n !} \sin (n \pi \gamma), \quad s \in(0, \infty)
\end{gathered}
$$


are the functions of Wright type, defined in $(0, \infty)$ which satisfies

$$
\zeta_{\gamma}(s) \geq 0, s \in(0, \infty), \quad \int_{0}^{\infty} \zeta_{\gamma}(s) d s=1,
$$

and

$$
\int_{0}^{\infty} s^{v} \zeta_{\gamma}(s) d s=\frac{\Gamma(1+v)}{\Gamma(1+\gamma v)}, \quad v \in(-1, \infty)
$$

For the definition of mild solution and the properties of operator families $\left\{S_{\gamma}(t)\right\}_{t \geq 0}$ and $\left\{P_{\gamma}(t)\right\}_{t \geq 0}$, and more detailed results, the references [2,16-18] are available.

From the Reference [18], we know that $\left\{S_{\gamma}(t)\right\}_{t \geq 0}$ and $\left\{P_{\gamma}(t)\right\}_{t \geq 0}$ are strongly continuous with $S_{\gamma}(t)$ and $P_{\gamma}(t)$ continuous in the uniform operator topology for $t>0$. From the reference [3], it implies that

$$
\begin{gathered}
S_{\gamma}^{\prime}(t) x=-t^{\gamma-1} A P_{\gamma}(t) x, \quad S_{\gamma}(t)=J_{t}^{1-\gamma}\left(t^{\gamma-1} P_{\gamma}(t)\right) \\
\left\|S_{\gamma}(t)\right\| \leq M, \quad\left\|P_{\gamma}(t)\right\| \leq \frac{M}{\Gamma(\gamma)}
\end{gathered}
$$

for $t>0, x \in X$, where the mark $J_{t}^{1-\gamma}$ expresses the Riemann-Liouville fractional integral of order $1-\gamma$ with respect to $t$, and

$$
\begin{gathered}
\left\|A^{\alpha} S_{\gamma}(t)\right\| \leq C_{\alpha, \gamma} t^{-\gamma \alpha}, \quad 0<\alpha<1,0<t<\infty, \\
\left\|A^{\alpha} P_{\gamma}(t)\right\| \leq C_{\alpha, \gamma} t^{-\gamma \alpha}, \quad 0<\alpha<2,0<t<\infty,
\end{gathered}
$$

where $C_{\alpha, \gamma}$ is a constant with respect to $\alpha$ and $\gamma$. The proof of the Theorem 5.1 in Reference [17] shows

$$
\left\|\frac{d}{d t}\left(t^{\gamma-1} A^{\alpha} P_{\gamma}(t)\right)\right\| \leq C_{\gamma} t^{\gamma(1-\alpha)-2},
$$

and combining this with the above inequality and the mean value theorems implies

$$
\begin{gathered}
t^{\gamma-1} A^{\alpha} P_{\gamma}(t)-s^{\gamma-1} A^{\alpha} P_{\gamma}(s)=\int_{s}^{t} \frac{d}{d \tau}\left(\tau^{\gamma-1} A^{\alpha} P_{\gamma}(\tau)\right) d \tau, \\
\left\|t^{\gamma-1} A^{\alpha} P_{\gamma}(t)-s^{\gamma-1} A^{\alpha} P_{\gamma}(s)\right\| \leq C_{\gamma} \int_{s}^{t} \tau^{\gamma(1-\alpha)-2} d \tau,
\end{gathered}
$$

where $C_{\gamma}$ is a constant.

Lemma 4. Suppose $0<\alpha<\beta \leq 1$ and $0<v=\beta-\alpha<1$. Then, for any $\varphi \in X_{\beta}$ and $h \in C([0, T], X)$, the mild solution $u$ of the LEE (5) has the regularity:

$$
u \in C^{\gamma v}\left([0, T], X_{\alpha}\right)
$$

and the map $H(\varphi, h)$ is continuous from $X_{\beta} \times C([0, T], X)$ to $C^{\gamma v}\left([0, T], X_{\alpha}\right)$.

Proof. Denote $v(t)=\int_{0}^{t}(t-s)^{\gamma-1} P_{\gamma}(t-s) h(s) d s$. When $\alpha>0$, for any $0 \leq t_{1}<t_{2} \leq T$, combining with Definition 1, (9), (10) and (12) we have

$$
\begin{aligned}
\left\|S_{\gamma}\left(t_{2}\right) \varphi-S_{\gamma}\left(t_{1}\right) \varphi\right\|_{\alpha} & =\left\|A^{\alpha} S_{\gamma}\left(t_{2}\right) \varphi-A^{\alpha} S_{\gamma}\left(t_{1}\right) \varphi\right\| \\
& =\left\|A^{-(\beta-\alpha)}\left(S_{\gamma}\left(t_{2}\right)-S_{\gamma}\left(t_{1}\right)\right) A^{\beta} \varphi\right\| \\
& \leq\left\|A^{-(\beta-\alpha)}\left(S_{\gamma}\left(t_{2}\right)-S_{\gamma}\left(t_{1}\right)\right)\right\| \cdot\|\varphi\|_{\beta}
\end{aligned}
$$




$$
\begin{aligned}
& =\left\|A^{-(\beta-\alpha)}\left(S_{\gamma}\left(t_{1}\right)\left(S_{\gamma}\left(t_{2}-t_{1}\right)-I\right)\right)\right\| \cdot\|\varphi\|_{\beta} \\
& =\left\|S_{\gamma}\left(t_{1}\right) A^{-(\beta-\alpha)}\left(S_{\gamma}\left(t_{2}-t_{1}\right)-I\right)\right\| \cdot\|\varphi\|_{\beta} \\
& =\left\|S_{\gamma}\left(t_{1}\right) \int_{0}^{t_{2}-t_{1}} s^{\gamma-1} A^{1-(\beta-\alpha)} P_{\gamma}(s) d s\right\| \cdot\|\varphi\|_{\beta} \\
& \leq M C_{\alpha, \gamma} \int_{0}^{t_{2}-t_{1}} s^{\gamma-1-\gamma(1-v)} d s \cdot\|\varphi\|_{\beta} \\
& \leq \frac{M C_{\alpha, \gamma}}{\gamma v}\left(t_{2}-t_{1}\right)^{\gamma v} \cdot\|\varphi\|_{\beta} .
\end{aligned}
$$

Here, we see that $S_{\gamma}(\cdot) \varphi \in C^{\gamma v}\left([0, T], X_{\alpha}\right)$, that is, $S_{\gamma}(t) \varphi$ is Hölder-continuous with exponent $\gamma v$. Next, we show that $v(t)$ is also Hölder-continuous. The previous proof implies

$$
\begin{aligned}
\left\|v\left(t_{2}\right)-v\left(t_{1}\right)\right\|_{\alpha} \leq & \| \int_{0}^{t_{1}} A^{\alpha}\left(\left(t_{2}-s\right)^{\gamma-1} P_{\gamma}\left(t_{2}-s\right)\right. \\
& \left.-\left(t_{1}-s\right)^{\gamma-1} P_{\gamma}\left(t_{1}-s\right)\right) h(s) d s \| \\
& +\left\|\int_{t_{1}}^{t_{2}} A^{\alpha}\left(\left(t_{2}-s\right)^{\gamma-1} P_{\gamma}\left(t_{2}-s\right)\right) h(s) d s\right\| \\
= & I_{1}+I_{2} .
\end{aligned}
$$

In view of the inequalities (13), (15) and the equality (14), we have

$$
\begin{aligned}
I_{1} \leq & \int_{0}^{t_{1}} \| A^{\alpha}\left(\left(t_{2}-s\right)^{\gamma-1} P_{\gamma}\left(t_{2}-s\right)\right. \\
& \left.-\left(t_{1}-s\right)^{\gamma-1} P_{\gamma}\left(t_{1}-s\right)\right)\|d s \cdot\| h \|_{C} \\
= & \left.\int_{0}^{t_{1}} \|\left(t_{2}-t_{1}+s\right)^{\gamma-1} A^{\alpha} P_{\gamma}\left(t_{2}-t_{1}+s\right)-s^{\gamma-1} A^{\alpha} P_{\gamma}(s)\right)\|d s \cdot\| h \|_{C} \\
= & \int_{0}^{t_{1}}\left\|\int_{s}^{t_{2}-t_{1}+s} \frac{d}{d \tau}\left(\tau^{\gamma-1} A^{\alpha} P_{\gamma}(\tau)\right) d \tau\right\| d s \cdot\|h\|_{C} \\
\leq & C_{\gamma} \int_{0}^{t_{1}} \int_{s}^{t_{2}-t_{1}+s} \tau^{\gamma(1-\alpha)-2} d \tau d s \cdot\|h\|_{C} \\
= & \frac{C_{\gamma}}{1-\gamma(1-\alpha)} \int_{0}^{t_{1}}\left(s^{\gamma(1-\alpha)-1}-\left(t_{2}-t_{1}+s\right)^{\gamma(1-\alpha)-1}\right) d s \cdot\|h\|_{C} \\
= & \frac{C_{\gamma}}{\gamma(1-\alpha)(1-\gamma(1-\alpha))}\left(t_{1}^{\gamma(1-\alpha)}-t_{2}^{\gamma(1-\alpha)}+\left(t_{2}-t_{1}\right)^{\gamma(1-\alpha)}\right) \cdot\|h\|_{C} \\
\leq & \frac{C_{\gamma}}{\gamma(1-\alpha)(1-\gamma(1-\alpha))}\left(t_{2}-t_{1}\right)^{\gamma(1-\alpha)} \cdot\|h\|_{C} \\
\leq & \frac{C_{\gamma}}{\gamma(1-\alpha)(1-\gamma(1-\alpha))}\left(t_{2}-t_{1}\right)^{\gamma v} \cdot\|h\|_{C},
\end{aligned}
$$

and

$$
\begin{aligned}
I_{2} & \leq \int_{t_{1}}^{t_{2}}\left\|\left(t_{2}-s\right)^{\gamma-1} A^{\alpha} P_{\gamma}\left(t_{2}-s\right)\right\| d s \cdot\|h\|_{C} \\
& \leq C_{\gamma} \int_{t_{1}}^{t_{2}} \int_{0}^{t_{2}-s} \tau^{\gamma(1-\alpha)-2} d \tau d s \cdot\|h\|_{C} \\
& =\frac{C_{\gamma}}{1-\gamma(1-\alpha)} \int_{t_{1}}^{t_{2}}\left(t_{2}-s\right)^{\gamma(1-\alpha)-1} d s \cdot\|h\|_{C} \\
& =\frac{C_{\gamma}}{\gamma(1-\alpha)(1-\gamma(1-\alpha))}\left(t_{2}-t_{1}\right)^{\gamma(1-\alpha)} \cdot\|h\|_{C}
\end{aligned}
$$




$$
\leq \frac{C_{\gamma}}{\gamma(1-\alpha)(1-\gamma(1-\alpha))}\left(t_{2}-t_{1}\right)^{\gamma v} \cdot\|h\|_{C}
$$

Therefore, $v(t)$ is also Hölder-continuous with exponent $\gamma v$. It follows that $u \in C^{\gamma v}\left([0, T], X_{\alpha}\right)$ and

$$
\begin{aligned}
\|H(\varphi, h)\|_{C^{\gamma v}\left([0, T], X_{\alpha}\right) \leq} & \frac{M C_{\alpha, \gamma}}{\gamma v}\left(t_{2}-t_{1}\right)^{\gamma v} \cdot\|\varphi\|_{\beta} \\
& +\frac{2 C_{\gamma}}{\gamma(1-\alpha)(1-\gamma(1-\alpha))}\left(t_{2}-t_{1}\right)^{\gamma v} \cdot\|h\|_{C} .
\end{aligned}
$$

Further, the map $H(\varphi, h)$ is continuous from $X_{\beta} \times C([0, T], X)$ to $C^{\gamma v}\left([0, T], X_{\alpha}\right)$.

Lemma 5. Let $\Omega$ be an open, bounded domain in $\mathbb{R}^{N}$ with $C^{2+\mu}$-smooth boundary $\partial \Omega$ and $p>\frac{N}{2}$. Then, for any $\varphi \in X_{\alpha}\left(\frac{N}{2 p}<\alpha<1\right)$ and $h \in F C^{\mu, \frac{\mu \gamma}{2}}(\bar{\Omega} \times[0, T])$, the LEE (5) possesses a unique classical solution:

$$
u \in C\left([0, T], X_{\alpha}\right) \cap F C^{2+\mu, \frac{2+\mu}{2}} \gamma(\bar{\Omega} \times(0, T]) .
$$

And it is also a classical solution of LFP (4).

Proof. Obviously, we have the continuous embedding

$$
F C^{\mu, \frac{\mu \gamma}{2}}(\bar{\Omega} \times[0, T]) \hookrightarrow F C^{\frac{\mu \gamma}{2}}([0, T], C(\bar{\Omega})) \hookrightarrow F C^{\frac{\mu \gamma}{2}}([0, T], X) .
$$

It is well-known ([6], Definition 2.1 and Theorem 4.1), when $\varphi \in X_{\alpha}$ and the $h$ is Hölder-continuous, the LEE (5) has a unique local classical solution:

$$
\left\{\begin{array}{l}
u \in C\left([0, T], X_{\alpha}\right) \cap C\left((0, T], X_{1}\right), \quad J_{t}^{1-\gamma} u \in C^{1}((0, T], X), \\
D_{t}^{\gamma} u \in C((0, T], X)
\end{array}\right.
$$

expressed by

$$
u(t)=S_{\gamma}(t) \varphi+\int_{0}^{t}(t-s)^{\gamma-1} P_{\gamma}(t-s) h(s) d s .
$$

Taking $\alpha^{\prime}=\frac{1}{2}\left(\alpha+\frac{N}{2 p}\right)$ and $\sigma=\alpha^{\prime}-\frac{N}{2 p}$. Then, from the Lemma 4 , the mild solutions of LEE (5) have the regularity:

$$
u \in C^{\gamma \sigma}\left([0, T], X_{\alpha^{\prime}}\right)
$$

and from (2) of Lemma 2, it follows that inclusion relation:

$$
\begin{aligned}
C^{\gamma \sigma}\left([0, T], X_{\alpha^{\prime}}\right) & \subset C^{\gamma \sigma}\left([0, T], C^{\sigma}(\bar{\Omega})\right) \subset C^{\frac{\gamma \sigma}{2}}\left([0, T], C^{\sigma}(\bar{\Omega})\right) \\
& \subset C^{\sigma, \frac{\gamma \sigma}{2}}(\bar{\Omega} \times[0, T]) \\
& =F C^{\sigma, \frac{\gamma \sigma}{2}}(\bar{\Omega} \times[0, T]) .
\end{aligned}
$$

For the case $\sigma<\mu$. We need the modified function $\phi_{\varepsilon} \in C^{2}([0, T],[0,1])$ defined by

$$
\phi_{\varepsilon}(t)= \begin{cases}0, & 0 \leq t \leq \frac{\varepsilon}{2} \\ 1, & \varepsilon \leq t \leq T\end{cases}
$$


where for any $\varepsilon \in(0, T]$. From (16), we see that

$$
\begin{aligned}
h_{1}= & \phi_{\varepsilon} h+u D_{t}^{\gamma} \phi_{\varepsilon}+\phi_{\varepsilon} D_{t}^{\gamma} u(0)-D_{t}^{\gamma}\left(\phi_{\varepsilon} u(0)\right) \\
& -\frac{\gamma}{\Gamma(1-\gamma)} \int_{0}^{t} \frac{(u(s)-u(t))\left(\phi_{\varepsilon}(s)-\phi_{\varepsilon}(t)\right)}{(t-s)^{\gamma+1}} d s-\frac{u \phi_{\varepsilon}}{\Gamma(1-\gamma) t \gamma} \\
= & \phi_{\varepsilon} h+u D_{t}^{\gamma} \phi_{\varepsilon}-\frac{\gamma}{\Gamma(1-\gamma)} \int_{0}^{t} \frac{(u(s)-u(t))\left(\phi_{\varepsilon}(s)-\phi_{\varepsilon}(t)\right)}{(t-s)^{\gamma+1}} d s-\frac{u \phi_{\varepsilon}}{\Gamma(1-\gamma) t^{\gamma}} \\
\in & F C^{\sigma, \frac{\gamma \sigma}{2}}(\bar{\Omega} \times[0, T]) .
\end{aligned}
$$

Corresponding to the function $h_{1}$, we consider the initial boundary value problems of parabolic type

$$
\left\{\begin{array}{l}
D_{t}^{\gamma} v(x, t)+A(x, D) v(x, t)=h_{1}, \quad(x, t) \in \Omega \times(0, T], \\
\left.v\right|_{\partial \Omega}=0, \quad t \in(0, T] \\
v(x, 0)=0, \quad x \in \Omega .
\end{array}\right.
$$

By Lemma 3, it is known that the problem (17) has a unique classical solution: $v \in$

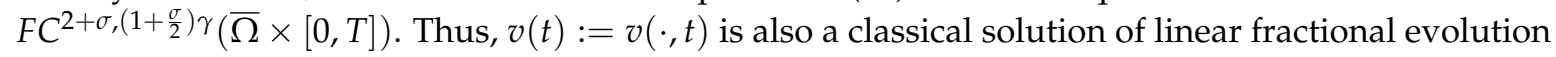
equations in $X$

$$
\left\{\begin{array}{l}
D_{t}^{\gamma} v(t)+A v(t)=h_{1}, \quad t \in(0, T], \\
v(0)=\theta .
\end{array}\right.
$$

where the $\theta$ is a zero element in Banach space $X$. Then, $\phi_{\varepsilon} u$ is a solution of problems (18).

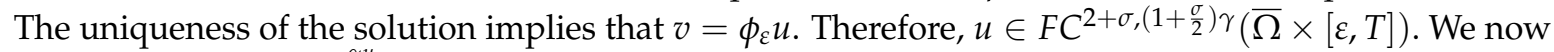
deduce that $h_{1} \in C^{\mu, \frac{\gamma \mu}{2}}(\bar{\Omega} \times[0, T])$. Again, repeating the above regularization process, we obtain

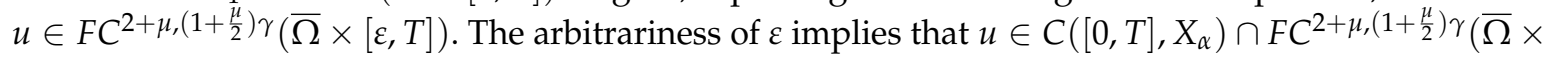
$(0, T])$ is a classical solution of LFP (4).

Setting $\sigma=\mu$ in the process of the proof above, we directly reach the same conclusion as above.

\section{Main Results}

Theorem 1. Let $\Omega$ be an open, bounded domain in $\mathbb{R}^{N}$ with $C^{2+\mu}$-smooth boundary $\partial \Omega(0<\mu<1)$. Suppose $p>N, \frac{1}{2}+\frac{N}{2 p}<\alpha<1$ and $\varphi \in X_{\alpha}$. If the nonlinear term $f: \bar{\Omega} \times \mathbb{R}^{+} \times \mathbb{R} \times \mathbb{R}^{N} \rightarrow \mathbb{R}$ is continuous and $f(x, t, \xi, \eta)$ is Hölder-continuous with exponent $\left(\mu, \frac{\gamma \mu}{2}\right)$ respect to $(x, t)$ and local Lipschitz-continuous with respect to $(\xi, \eta)$. Then, the FPDE (1)-(3) possesses a unique saturated classical solution:

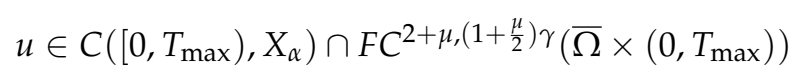

and

$$
\varlimsup_{t \rightarrow T_{\max }^{-}}\|u(\cdot, t)\|_{C^{1}(\Omega)}=+\infty
$$

for $0<T_{\max }<+\infty$, where the $X_{\alpha}=\left(D\left(A^{\alpha}\right),\|\cdot\|_{\alpha}\right)$ is the interpolation space between $X_{0}$ and $X_{1}$.

Proof. Denote $E=C^{1}(\bar{\Omega})$. As a result of $p>N$ and $\frac{1}{2}+\frac{N}{2 p}<\alpha<1$, then, according to (1) of Lemma 2,

$$
X_{\alpha} \hookrightarrow E \hookrightarrow X .
$$

Owing to the assumption that the nonlinear term $f(x, t, \xi, \eta)$ is Hölder-continuous with respect to $(x, t)$ and local Lipschitz-continuous with respect to $(\xi, \eta)$, we define the mapping as

$$
\begin{gathered}
F: \mathbb{R}^{+} \times E \rightarrow X ; \\
F(t, v)(\cdot)=f(\cdot, t, v(\cdot, t), \nabla v(\cdot, t)) .
\end{gathered}
$$


Then, the problem (1)-(3) can be rewritten as the following initial value problems of the evolution equations with sectorial operator (IVPS)

$$
\left\{\begin{array}{l}
D_{t}^{\gamma} u(t)+A u(t)=F(t, u(t)), \quad t>0, \\
u(0)=\varphi \in X_{\alpha} .
\end{array}\right.
$$

It is well-known ([6], Definition 2.1 and Theorem 4.1) that the basic theory of the evolution equations with sectorial operator implies the IVPS (19) possesses a unique local solution:

$$
\left\{\begin{array}{l}
u \in C\left([0, T], X_{\alpha}\right) \cap C\left((0, T], X_{1}\right), \quad J_{t}^{1-\gamma} u \in C^{1}((0, T], X), \\
D_{t}^{\gamma} u \in C((0, T], X) .
\end{array}\right.
$$

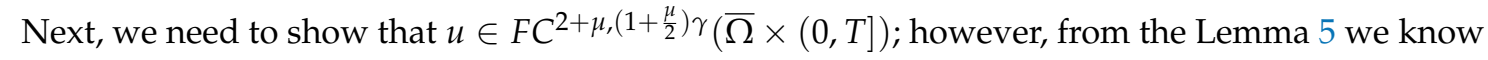
that it is obvious. Similar to the Reference [1], applied to piecewise extended method for IVPS (19), we easily obtain the FPDE (1)-(3) which possesses a unique saturated classical solution:

$$
u \in C\left(\left[0, T_{\max }\right), X_{\alpha}\right) \cap F C^{2+\mu,\left(1+\frac{\mu}{2}\right) \gamma}\left(\bar{\Omega} \times\left(0, T_{\max }\right)\right)
$$

and

$$
\varlimsup_{t \rightarrow T_{\max }^{-}}\|u(\cdot, t)\|_{C^{1}(\Omega)}=+\infty
$$

for $0<T_{\max }<+\infty$, where the $X_{\alpha}=\left(D\left(A^{\alpha}\right),\|\cdot\|_{\alpha}\right)$ is the interpolation space between $X_{0}$ and $X_{1}$. This completes the proof of Theorem 1 .

Theorem 2. Let $\Omega$ be an open, bounded domain in $\mathbb{R}^{N}$ with $C^{2+\mu}$-smooth boundary $\partial \Omega(0<\mu<1), p>N$, $\varphi \in C_{0}^{2}(\bar{\Omega})$ and $\varphi \geq 0$, the nonlinear term $f: \bar{\Omega} \times \mathbb{R}^{+} \times \mathbb{R} \times \mathbb{R}^{N} \rightarrow \mathbb{R}$ is continuous and $f(x, t, \bar{\xi}, \eta)$ is Hölder-continuous with exponent $\left(\mu, \frac{\gamma \mu}{2}\right)$ respect to $(x, t)$ and local Lipschitz-continuous with respect to $(\xi, \eta)$. If $f$ satisfies the following conditions

(F1) there exist constants $a=a(T)>0$ and $b=b(T)>0$ for any $T>0$ such that

$$
0 \leq f(x, t, \xi, \eta) \leq a \xi+b
$$

for all $(x, t, \xi, \eta) \in \bar{\Omega} \times[0, T] \times[0,+\infty) \times \mathbb{R}^{N}$;

(F2) there exist constants $c=c(T, R)>0$ and $\rho=\rho(T, R) \in[1,2)$ for any $T, R>0$ such that $f$ satisfies

$$
|f(x, t, \xi, \eta)| \leq c\left(1+|\eta|^{\rho}\right)
$$

for all $(x, t, \xi, \eta) \in \bar{\Omega} \times[0, T] \times[-R, R] \times \mathbb{R}^{N}$.

Then the FPDE (1)-(3) possesses a unique positive global solution:

$$
\left\{\begin{array}{l}
u \in C^{1,0}(\bar{\Omega} \times[0,+\infty)), \quad J_{t}^{1-\gamma} u \in C^{2,1}(\bar{\Omega} \times(0,+\infty)), \\
D_{t}^{\gamma} u \in C(\bar{\Omega} \times(0,+\infty)) .
\end{array}\right.
$$

Proof. Denote $E=C^{1}(\bar{\Omega})$. Thanks to $p>N$, choose $\frac{1}{2}+\frac{N}{2 p}<\alpha<1$ and according to the (1) of Lemma 2, we know that

$$
X_{\alpha} \hookrightarrow E \hookrightarrow X .
$$

Noticing $C_{0}^{2}(\bar{\Omega}) \subset D(A) \subset X_{1} \subset X_{\alpha}$, then $\varphi \in X_{\beta}(\alpha<\beta \leq 1)$. Using Theorem 1 to $\varphi \in X_{\beta}(\alpha<\beta \leq$ $1)$, then the FPDE (1)-(3) possesses a unique local classical solution: 


$$
\left\{\begin{array}{l}
u \in C\left([0, T], X_{\alpha}\right), \quad J_{t}^{1-\gamma} u \in C^{2,1}(\bar{\Omega} \times(0, T]), \\
D_{t}^{\gamma} u \in C((0, T], X),
\end{array}\right.
$$

and when $0<T<+\infty$,

$$
\varlimsup_{t \rightarrow T^{-}}\|u(t)\|_{C^{1}(\Omega)}=+\infty .
$$

Our goal is to prove $T=+\infty$. If $T=+\infty$ is not true, then $T<+\infty$. Now, we show that

$$
\|u(t)\|_{C^{1}(\Omega)} \leq c_{0}^{*}, \quad t \in[0, T),
$$

where $c_{0}^{*}$ is a constant. We divide this into two steps to prove that the (22) is held.

Step 1: When $\varphi \geq 0$, the solution of FPDE (1)-(3) is positive.

For any $T_{1} \in(0, T)$, the solution of FPDE (1)-(3) satisfies

$$
\left\{\begin{array}{l}
u \in C^{1,0}\left(\bar{\Omega} \times\left[0, T_{1}\right]\right), \quad J_{t}^{1-\gamma} u \in C^{2,1}\left(\bar{\Omega} \times\left(0, T_{1}\right]\right), \\
D_{t}^{\gamma} u \in C\left(\bar{\Omega} \times\left(0, T_{1}\right]\right) .
\end{array}\right.
$$

For the constants $a$ and $b$ corresponding to $T$ in the assumption $(F 1)$, let us consider the initial boundary problems

$$
\left\{\begin{array}{l}
D_{t}^{\gamma} u(x, t)+A(x, D) u(x, t)=a u(x, t)+b, \quad(x, t) \in \Omega \times(0, T] \\
\left.u\right|_{\partial \Omega}=0, \quad t \in(0, T] \\
u(x, 0)=\varphi(x), \quad x \in \Omega .
\end{array}\right.
$$

Obviously, the above problems (23) possess a classical solution:

$$
\left\{\begin{array}{l}
w \in C\left([0, T], X_{\alpha}\right), \quad J_{t}^{1-\gamma} w \in C^{2,1}(\bar{\Omega} \times(0, T]), \\
D_{t}^{\gamma} w \in C((0, T], X) .
\end{array}\right.
$$

By virtue of the strong maximum principle and the comparison principle from the Reference [7] and the assumption $(F 1)$, we know that $w \geq 0$ for $(x, t) \in \bar{\Omega} \times[0, T]$ and

$$
0 \leq u(x, t) \leq w(x, t), \quad(x, t) \in \bar{\Omega} \times[0, T] .
$$

Further, the inequality (24) is held, and

$$
\|u(t)\|_{C(\bar{\Omega})} \leq\|w(t)\|_{C(\bar{\Omega})} \leq R
$$

Step 2: $C^{1}(\bar{\Omega})$-estimate.

We declare the $c_{i}^{*}(i=1,2, \ldots)$ is a constant where the latter has a different process of proof.

Let $c=c(T, R)>0$ and $\rho=\rho(T, R) \in(1,2)$ be two constants in the assumption (F2), where $T$ and $R$ correspond to (25). Then,

$$
|f(x, t, u(x, t), \nabla u(x, t))| \leq c\left(1+|\nabla u(x, t)|^{\rho}\right), \quad(x, t) \in \bar{\Omega} \times[0, T] .
$$

We set $p>\frac{N \rho}{2-\rho}(>N)$. Note that $\frac{1}{2}+\frac{N}{2 p}<\frac{1}{\rho}$, with the choice of $\frac{1}{2}+\frac{N}{2 p}<\alpha<\frac{1}{\rho}$. We define the mapping

$$
\begin{gathered}
F: \mathbb{R}^{+} \times X_{\alpha} \rightarrow X ; \\
F(t, v)(\cdot)=f(\cdot, t, v(\cdot, t), \nabla v(\cdot, t)) .
\end{gathered}
$$


Then, from the assumption (F2), (26) and (1) of Lemma 2 for $u \in C\left([0, T], X_{\alpha}\right)$, we have

$$
\begin{aligned}
\|F(t, u(t))\| & \leq\left(\int_{\Omega}|F(t, u(t))|^{p} d x\right)^{\frac{1}{p}} \\
& \leq\left(\int_{\Omega}\|F(t, u(t))\|^{p} d x\right)^{\frac{1}{p}} \\
& \leq|\Omega|^{\frac{1}{p}}\|F(t, u(t))\|_{C(\bar{\Omega})} \\
& \leq c|\Omega|^{\frac{1}{p}}\left(1+\|\nabla u(t)\|_{C(\bar{\Omega})}^{\rho}\right) \\
& \leq c_{1}^{*}\left(1+\|u(t)\|_{C^{1}(\bar{\Omega})}^{\rho}\right) \\
& \leq c_{2}^{*}\left(1+\|u(t)\|_{\alpha}^{\rho}\right), \quad t \in[0, T] .
\end{aligned}
$$

where the $|\Omega|$ is the volume of the $\Omega$. Introducing $\alpha_{1}=\alpha \rho \in(\alpha, 1)$, from Lemma 1 and (25), we obtain

$$
\begin{aligned}
\|u(t)\|_{\alpha}^{\rho} & =\|u(t)\|_{\alpha}^{\frac{\alpha_{1}}{\alpha}} \\
& \leq 2 c_{3}^{*}\|u(t)\|_{\alpha_{1}}\|u(t)\|_{0}^{\frac{\alpha_{1}}{\alpha}-1} \\
& \leq 2 c_{3}^{*}\|u(t)\|_{\alpha_{1}}\|u(t)\|_{0}^{\rho-1} \\
& \leq 2 c_{3}^{*}|\Omega|^{\frac{\rho-1}{p}}\|u(t)\|_{\alpha_{1}}\|u(t)\|_{C(\bar{\Omega})}^{\rho-1} \\
& \leq c_{4}^{*}\|u(t)\|_{\alpha_{1}} .
\end{aligned}
$$

Combining this with (27) and (28), it is clear that

$$
\|F(t, u(t))\| \leq c_{5}^{*}\left(1+\|u(t)\|_{\alpha_{1}}\right) .
$$

Observe that $u \in C\left([0, T], X_{\alpha}\right)$ satisfies

$$
u(t)=S_{\gamma}(t) \varphi+\int_{0}^{t}(t-s)^{\gamma-1} P_{\gamma}(t-s) F(s, u(s)) d s .
$$

Making the norm- $\|\cdot\|_{\alpha_{1}}$ for the above two sides of the equality, we see that

$$
\begin{aligned}
\|u(t)\|_{\alpha_{1}} & \leq\left\|A^{\alpha_{1}} S_{\gamma}(t) \varphi\right\|+\int_{0}^{t}(t-s)^{\gamma-1}\left\|A^{\alpha_{1}} P_{\gamma}(t-s)\right\|\|F(s, u(s))\| d s \\
& \leq\left\|S_{\gamma}(t)\right\|\left\|A^{\alpha_{1}} \varphi\right\|+c_{5}^{*} C_{\alpha_{1}, \gamma} \int_{0}^{t}(t-s)^{\gamma-\gamma \alpha_{1}-1}\left(1+\|u(s)\| \alpha_{\alpha_{1}}\right) d s .
\end{aligned}
$$

The above inequality implies

$$
\|u(t)\|_{\alpha_{1}} \leq c_{6}^{*}+c_{7}^{*} \int_{0}^{t}(t-s)^{\gamma-\gamma \alpha_{1}-1}\|u(s)\|_{\alpha_{1}} d s .
$$

Applying Gronwall's inequality for the $\bar{\phi}(t)=\|u(t)\|_{\alpha_{1}}$ of (29), we have

$$
\|u(t)\|_{\alpha_{1}} \leq c_{9}^{*} c_{8}^{*} .
$$

Due to $\alpha<\alpha_{1}<1$ and $X_{\alpha_{1}} \hookrightarrow X_{\alpha} \hookrightarrow C^{1}(\bar{\Omega})$, it follows that

$$
\|u(t)\|_{C^{1}(\bar{\Omega})} \leq c_{10}^{*}\|u(t)\|_{\alpha_{1}} \leq c_{11}^{*},
$$

which contradicts with (21). Therefore, $T=+\infty$. Namely, then the FPDE (1)-(3) possesses a unique positive global solution: 


$$
\left\{\begin{array}{l}
u \in C^{1,0}(\bar{\Omega} \times[0,+\infty)), \quad J_{t}^{1-\gamma} u \in C^{2,1}(\bar{\Omega} \times(0,+\infty)), \\
D_{t}^{\gamma} u \in C(\bar{\Omega} \times(0,+\infty)) .
\end{array}\right.
$$

For case: $\rho=1$, the conclusion of Theorem 2 is obvious.

Remark 1. In the assumption $F(2)$, the $f(x, t, \xi, \eta)$ allows for superlinear growth on $\eta$.

\section{Applications}

Using the result obtained in Section 3, we can solve the initial boundary value problem to the following semi-linear sub-diffusion with gradient terms

$$
\left\{\begin{array}{l}
D_{t}^{\gamma} u(x, t)-\Delta u(x, t)=\frac{e^{-t}}{1+|u(x, t)|}+|\nabla u|^{\frac{3}{2}}, \quad x \in \Omega, t \geq 0 \\
\left.u\right|_{\partial \Omega}=0, \\
u(x, 0)=\varphi(x), \quad x \in \bar{\Omega}
\end{array}\right.
$$

where $D_{t}^{\gamma}$ is the regularized Caputo's fractional derivative of order $0<\gamma \leq 1, \Delta$ is the Laplace operator, $\nabla$ is the Gradient operator, $\Omega \subset \mathbb{R}^{N}$ with $C^{2+\mu}$-smooth boundary $\partial \Omega(0<\mu<1), \varphi(x) \in C_{0}^{2}(\bar{\Omega})$ and $\varphi(x) \geq 0$.

Let $E=L^{2}(\bar{\Omega})$ with the norm $\|\cdot\|_{2}$. We define an operator $A$ in Banach space $E$ by

$$
A u=-\Delta u, \quad D(A)=H^{2}(\Omega) \cap H_{0}^{1}(\Omega),
$$

where $H^{2}(\Omega)$ is the completion of the space $C^{2}(\Omega)$ with respect to the norm

$$
\|u\|_{H^{2}(\Omega)}=\left(\int_{\Omega} \sum_{|\vartheta| \leq 2}\left|D^{\vartheta} u(x)\right|^{2} d x\right)^{\frac{1}{2}}
$$

$C^{2}(\Omega)$ is the set of all continuous functions defined on $\Omega$ which have continuous partial derivatives of order less than or equal to $2, H_{0}^{1}(\Omega)$ is the completion of $C^{2}(\Omega)$ with respect to the norm $\|u\|_{H^{1}(\Omega)}$, and $C_{0}^{1}(\Omega)$ is the set of all functions $u \in C^{1}(\Omega)$ with compact supports on the domain $\Omega$. It is well-known from [14] that $-A$ generates a uniformly bounded analytic semigroup $T(t)(t \geq 0)$ in $E$. From the definition of nonlinear term $f$, we can easily verify that the assumptions (F1) and (F2) are satisfied with $a=1, b=\max _{(x, t) \in \bar{\Omega} \times[0, \infty)}|\nabla u(x, t)|^{\frac{3}{2}}$ and $c=1, \rho=\frac{3}{2}$. Therefore, by Theorem 2, we have the following existence result.

Theorem 3. The initial boundary value problem to the following semi-linear sub-diffusion with gradient terms (30) has a positive global solution

$$
\left\{\begin{array}{l}
u \in C^{1,0}(\bar{\Omega} \times[0,+\infty)), \quad J_{t}^{1-\gamma} u \in C^{2,1}(\bar{\Omega} \times(0,+\infty)), \\
D_{t}^{\gamma} u \in C(\bar{\Omega} \times(0,+\infty)) .
\end{array}\right.
$$

It should be pointed out that this conclusion cannot be obtained from the known results of References $[1-6,8,17]$.

\section{Conclusions}

In this work, existence and uniqueness of saturated classical solutions and the positive classical solutions to initial boundary value problems of the semi-linear fractional parabolic partial differential equations with gradient terms was obtained. Furthermore, the results obtained in this paper are a supplement to the existing literature and essentially extends some existing results in this area. Our future work will be devoted to formulating conditions that guarantee the existence and uniqueness 
of classical solutions and the positive classical solutions for initial boundary value problems of semi-linear sub-diffusion with gradient terms.

Author Contributions: Y.G. and Y.L. carried out the first draft of this manuscript, Y.G. prepared the final version of the manuscript. All authors have read and agreed to the published version of the manuscript.

Funding: The research is supported by the National Natural Science Function of China (No. 11661071).

Acknowledgments: The authors would like to thank the anonymous reviewers for their valuable comments and suggestions.

Conflicts of Interest: The author declares no conflict of interest.

\section{References}

1. Chen, P.; Li, Y.; Chen, Q.; Feng, B. On the initial value problem of fractional evolution equations with noncompact semigroup. Comput. Math. Appl. 2014, 67, 1108-1115. [CrossRef]

2. Chen, P.; Li, Y. Existence of mild solutions for fractional evolution equations with mixed monotone non-local conditions. Z. Angew. Math. Phys. 2014, 65, 711-728. [CrossRef]

3. $\mathrm{Mu}, \mathrm{J} . ;$ Ahmad, B.; Huang, S. Existence and regularity of solutions to time-fractional diffusion equations. Comput. Math. Appl. 2017, 73, 985-996. [CrossRef]

4. Chen, P.; Zhang, X.; Li, Y. Cauchy problem for fractional non-autonomous evolution equations. Banach J. Math. Anal. 2020, 14, 559-584. [CrossRef]

5. Chen, P.; Zhang, X.; Li, Y. Existence and approximate controllability of fractional evolution equations with non-local conditions via resolvent operators. Fract. Calc. Appl. Anal. 2020, 23, 268-291. [CrossRef]

6. El-Borai, M.M.; Debbouche, A. On some fractional integro-differential equations with analytic semigroups. Int. J. Contemp. Math. Sci. 2009, 4, 1361-1371.

7. Luchko, Y. Maximum principle for the generalized time-fractional diffusion equation. J. Math. Anal. Appl. 2009, 351, 218-223. [CrossRef]

8. Zhang, X.; Chen, P.; Li, Y. Fractional retarded differential equations involving mixed non-local plus local initial conditions. Numer. Funct. Anal. Optim. 2019, 40, 1678-1702. [CrossRef]

9. Kilbas, A.A.; Srivastava, H.M.; Trujillo, J.J. Theory and Applications of Fractional Differential Equations; North-Holland Mathematics Studies; Elsevier Science B.V.: Amsterdam, The Netherlands, 2006, Volume 204.

10. Adams, R.A.; Fournier, J.J.F. Sobolev Spaces, 2nd ed.; Academic Press: Amsterdam, The Netherlands, 2003.

11. Evans, L.C. Partial Differential Equations, 2nd ed.; American Mathematical Society: Providence, RI, USA, 2010.

12. Henry, D. Geometric Theory of Semi-Linear Parabolic Equations; Lecture Notes in Math; Springer: New York, NY, USA, 1981; Volume 840.

13. Krasnoschok, M.; Vasylyeva, N. On a solvability of a nonlinear fractional reaction-diffusion system in the Hölder spaces. Nonlinear Stud. 2013, 20, 591-621.

14. Pazy, A. Semigroups of Linear Operators and Applications to Partial Differential Equations; Springer: Berlin, Germany, 1983.

15. Carracedo, C.M.; Alix, M.S. The Theory of Fractional Powers of Operators; Elsevier: North-Holland Mathematics Studies, 187; North-Holland Publishing Co.: Amsterdam, The Netherlands, 2001.

16. Wang, R.N.; Chen, D.H.; Xiao, T.J. Abstract fractional Cauchy problems with almost sectorial operators. J. Differ. Equ. 2012, 252, 202-235. [CrossRef]

17. Zhou, Y.; Peng, L. On the time-fractional Navier-Stokes equations. Comput. Math. Appl. 2017, 73, 874-891. [CrossRef]

18. Zhou, Y.; Wang, J.; Zhang, L. Basic theory of Fractional Differential Equations, 2nd ed.; World Scientific: Singapore, 2017.

(c) 2020 by the authors. Licensee MDPI, Basel, Switzerland. This article is an open access article distributed under the terms and conditions of the Creative Commons Attribution (CC BY) license (http://creativecommons.org/licenses/by/4.0/). 